\title{
Properties of Complemented Semirings
}

\author{
G. Mallikarjuna ${ }^{1}$, Dr. G. Shobhalatha ${ }^{2}$ \\ ${ }^{1}$ Research scholar, Dept. of Mathematics, S.K.University, Ananthapuramu- (A.P.), India. \\ ${ }^{2}$ Professor, Dept. of Mathematics, S.K.University, Ananthapuramu- (A.P.), India.
}

\begin{abstract}
Semiring theory is one of the most developing branch of Mathematics with wide application in many disciplines such as Computer science, Coding theory, Topological space and many researchers studies different structure of semirings like Boolean like semirings, ternary semirings, complemented ternary semirings, gamma semirings, Complemented semirings etc. In this paper, we discuss some properties of Complemented semirings. We determine the additive and multiplicative structures of Complemented semirings by assuming different properties on the additive (multiplicative) structures.
\end{abstract}

Keywords: Band, left singular, right singular, multiplicatively sub idempotent, commutative, rectangular band.

\section{Introduction}

Historically semirings first appear implicitly in Dedekind and later in Macaulay, Noether and Lorenzo in connection with the study of a ring. However semirings first appear explicitly in Vandiver, also in connection with the axiomatization of arithmetic of natural numbers. Semirings have been studied by various researchers in an attempt to broaden techniques coming from semigroup theory or ring theory or in connection with applications.

However in semirings it is possible to derive the additive structures from their special multiplicative structures and vice versa. The semiring identities are taken from the book of Jonathan S. Golan, entitled " semirings and their Applications". In this paper we investigate the additive and multiplicative properties of Complemented semi rings.

\section{Preliminaries}

Definition2.1: A Triple $(S,+,$.$) is said to be a semiring if S$ is a non-empty set and '+' and ' ' are binary operations on $S$ satisfying that

i. $(S,+)$ is a semigroup

ii. $(S,$.$) is a semigroup$

iii. $a(b+c)=a b+a c$ and $(b+c) a=b a+c a$ for all $a, b, c$ in $S$.

Definition 2.2: An element ' $a$ ' of a semiring $S$ is complemented if there exists an element 'b' in $S$ satisfying $a+b=1$ and $a b=b a=0$ for all $a$, in $S$.

Definition 2.3: An element ' $a$ ' of a semiring $S$ is multiplicatively sub idempotent if $a+a^{2}=a$ and $S$ is multiplicatively sub idempotent if each of its element is multiplicatively sub idempotent.

Definition 2.4: A Semigroup $(S,+)$ is said to be a band if it satisfies the identity $a+a=a$ for all $a$ in $S$.

Definition 2.5: A semigroup $(S,$.$) is said to be a rectangular band if it satisfies the identity a b a=a$ for all $a, b$ in $S$.

Definition 2.6: A Semigroup $(S,$.$) is said to be a left (right) singular if it satisfies the identity a b=a(a \mathrm{~b}=\mathrm{b})$ for all $a, b$ in $S$.

\section{Mainresults}

Theorem 3.1: Let $(S,+,$.$) be a complemented semiring. If (S,+)$ is commutative, then $a+1=1+a=a$ if and only if $(S,+)$ is a rectangular band.

Proof: Let $(S,+,$.$) be a complemented semiring and let (S,+)$ be a commutative, that is $a+b=b+a$ for all $a, b \in S$.

Since $S$ complemented semiring, we have $a+b=1$ and $a b=b a=0$ for all $a, b \in S$.

$$
\Rightarrow b+a=1 \quad \text { ( since }(S,+) \text { is commutative) }
$$


Adding ' $a$ ' on both sides, we get

$\Rightarrow a+b+a=a+1$

$\Rightarrow a=a+1 \quad$ ( since $(S,+)$ is rectangular band)

Again for $a+b=1$ and $a b=b a=0$

Adding ' $a$ ' on both sides, we get

$$
\begin{aligned}
\Rightarrow & a+b+a=1+a \\
\Rightarrow & a=1+a
\end{aligned}
$$

Therefore from (1) \& (2) $a+1=1+a=a$

Conversely

We have $a+b=1$ and $a b=b a=0$ for all $a, b \in S$.

$$
\Rightarrow b+a=1 \quad \text { ( since }(S,+) \text { is commutative) }
$$

Adding ' $a$ ' on both sides, we get

$$
\begin{aligned}
& \Rightarrow a+b+a=a+1 \\
& \Rightarrow a+b+a=a \quad(\text { since } a=a+1)
\end{aligned}
$$

Therefore $(S,+)$ is a rectangular band.

Theorem 3.2: Let $(S,+,$.$) be a complemented semiring containing the multiplicative identity 1$. If $S$ contains an additive identity zero.Then $(S,$.$) is a band.$

Proof: Let $(S,+,$.$) be a complemented semiring, and let '1' be the multiplicative identity of S$.

Since $a+b=1$ and $a b=b a=0$ for all $a, b \in S$,

By Theorem 3.1, we have $a+1=a$

Adding ' $\mathrm{b}$ ' on both sides, we get

$$
\begin{aligned}
& \Rightarrow a+1+b=a+b \\
& \Rightarrow a+1+b=1
\end{aligned}
$$

Now consider $a+1+b=1$ and $a b=b a=0$

Multiplying with ' $a$ ' on both sides, we get

$$
\begin{aligned}
& a(a+1+b)=a 1 \\
& \Rightarrow \quad a a+a 1+a b=a \\
& \Rightarrow a^{2}+a+a b=a \\
& \Rightarrow a^{2}+a+0=a \\
& \Rightarrow a^{2}+a=a \\
& \Rightarrow a(a+1)=a \\
& \Rightarrow a a=a \\
& \Rightarrow a^{2}=a
\end{aligned}
$$

Therefore $(S,$.$) is a band.$

Example 1: Consider the set $S=\{1, a\}$. We define additive and multiplicative structure on $S$ as shown in the following tables.
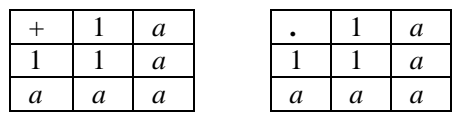

Then $S$ is a complemented semiring satisfies all conditions of the above theorem and $(S,$.$) is a band.$

Example 2: Consider the set $S=\{0,1, a, b\}$. We define additive and multiplicative structure on $S$ as shown in the following tables.

\begin{tabular}{|c|c|c|c|c|}
\hline+ & 0 & 1 & $a$ & $\mathrm{~b}$ \\
\hline 0 & 0 & 1 & $a$ & $\mathrm{~b}$ \\
\hline 1 & 1 & 1 & $a$ & $\mathrm{~b}$ \\
\hline$a$ & $a$ & $a$ & $a$ & 1 \\
\hline $\mathrm{b}$ & $\mathrm{b}$ & $\mathrm{b}$ & 1 & $\mathrm{~b}$ \\
\hline
\end{tabular}

\begin{tabular}{|c|c|c|c|c|}
\hline. & 0 & 1 & $a$ & $\mathrm{~b}$ \\
\hline 0 & 0 & 0 & 0 & 0 \\
\hline 1 & 0 & 1 & $a$ & $\mathrm{~b}$ \\
\hline$a$ & 0 & $a$ & $a$ & 0 \\
\hline $\mathrm{b}$ & 0 & $\mathrm{~b}$ & 0 & $\mathrm{~b}$ \\
\hline
\end{tabular}

Then $(S,$.$) is a band.$ 
Theorem 3.3: Let $(S,+,$.$) be a complemented semiring containing the multiplicative identity 1$. If $(S,$.$) is a$ band, then (i) $(S,+)$ is singular, (ii) $(S,+)$ is commutative, and (iii) $(S,+)$ is a rectangular band.

Proof: Let $(S,+,$.$) be a complemented semiring and let '1' be a multiplicative identity of S$, and $(S,$.$) is a$ band, that is $a^{2}=a$ for all $a \in S$.

Since $S$ complemented semiring, wehave $a+b=1$ and $a b=b a=0$ forall $a, b \in S$.

(i) By Theorem 3.1, we have $a+1=a$

Adding ' $\mathrm{b}$ ' on both sides, we get

$$
\begin{aligned}
& \Rightarrow a+1+b=a+b \\
& \Rightarrow a+1+b=1 \quad \text { From (1) }
\end{aligned}
$$

Now consider $a+1+b=1$ and $a b=b a=0$ for all $a, b \in S$.

Multiplying with ' $a$ ' on both sides, we get

$$
\begin{aligned}
& a(a+1+b)=a 1 \\
& \Rightarrow a a+a 1+a b=a \\
& \Rightarrow \quad a^{2}+a+a b=a \\
& \Rightarrow \quad a^{2}+a(1+b)=a \\
& \Rightarrow \quad a^{2}+a b=a
\end{aligned}
$$

(By theorem 3.1, i.e. $1+b=b$ )

Adding ' $\mathrm{b}$ ' on both sides, we get

$$
\begin{aligned}
& \Rightarrow a^{2}+a b+b=a+b \\
& \Rightarrow a^{2}+(a+1) b=a+b \\
& \Rightarrow \quad a^{2}+a b=a+b \\
& \Rightarrow a^{2}+0=a+b \\
& \text { From (2) } \\
& \Rightarrow a^{2}=a+b \\
& \Rightarrow a=a+b
\end{aligned}
$$

Therefore $(S,+)$ is left singular.

Now consider $a+1+b=1$ and for all $a, b \in S$. (by theorem 3.1)

Multiplying with ' $b$ ' on both sides, we get

$$
\begin{aligned}
& b(a+1+b)=b 1 \\
\Rightarrow & b a+b+b b=b \\
\Rightarrow & b a+b+b^{2}=b \\
\Rightarrow & b(a+1)+b^{2}=b
\end{aligned}
$$

$\Rightarrow b a+b^{2}=b$

(by theorem 3.1)

Adding ' $a$ ' on both sides, we get

$$
\begin{aligned}
& \Rightarrow \quad a+b a+b^{2}=a+b \\
& \Rightarrow \quad(1+b) a+b^{2}=a+b \\
& \Rightarrow \quad b a+b^{2}=a+b \\
& \Rightarrow 0+b^{2}=a+b \\
& \Rightarrow b^{2}=a+b \\
& \Rightarrow b=a+b \\
& \Rightarrow(S,+) \text { is right singular }
\end{aligned}
$$

(since $(S,$.$) is a band)$

Therefore $(S,+)$ is singular

(ii) Consider $a b+b+b^{2}+a^{2}+a+a b=(a+1+b) b+a(a+1+b)$

$$
\begin{aligned}
& \Rightarrow \quad 0+b+b^{2}+a^{2}+a+0=(a+1+b)(b+a) \\
& \Rightarrow \quad b(1+b)+a(a+1)=a(b+a)+1(b+a)+b(b+a) \\
& \Rightarrow \quad b b+a a=a b+a^{2}+b+a+b^{2}+b a \quad \text { (by theorem 3.1) }
\end{aligned}
$$




$$
\begin{array}{lll}
\Rightarrow & b^{2}+a^{2}=a b+a^{2}+b+a+b^{2}+b a & \\
\Rightarrow & b+a=0+a+b+a+b+b a & (\text { since }(S, .) \text { is a band, and } a \mathrm{~b}=0) \\
\Rightarrow & b+a=a+b+a+b(1+a) & \\
\Rightarrow & b+a=a+b+a+b a & \text { (by theorem 3.1) } \\
\Rightarrow & b+a=a+b+(1+b) a & \\
\Rightarrow & b+a=a+b+b a & \text { (by theorem 3.1) } \\
\Rightarrow & b+a=a+b+0 & \quad \text { (since ba=0) } \\
\Rightarrow & b+a=a+b &
\end{array}
$$

Therefore $(S,+)$ is commutative.

(iii) Now consider $a+1+b=1$ for all $a, b \in S$. (by theorem 3.1).

Multiplying with ' $b$ ' on both sides, we get

$$
\begin{gathered}
b(a+1+b)=b 1 \\
\Rightarrow \quad b a+b+b b=b \\
\Rightarrow \quad b a+b+b^{2}=b \\
\Rightarrow \quad b(a+1)+b^{2}=b
\end{gathered}
$$

$\Rightarrow b a+b^{2}=b$

(by theorem 3.1)

Adding ' $a$ ' on both sides, we get

$$
\begin{aligned}
& \Rightarrow a+b a+b^{2}=a+b \\
& \Rightarrow \quad(1+b) a+b^{2}=a+b \\
& \Rightarrow b a+b^{2}=a+b \quad \text { (by theorem 3.1) } \\
& \Rightarrow b a+b=a+b \quad \text { (since }(S, .) \text { is a band) } \\
& \Rightarrow b(a+1)=a+b \\
& \Rightarrow b a=a+b \quad \text { (by theorem 3.1) }
\end{aligned}
$$

Adding ' $a$ ' on both sides, we get

$$
\begin{aligned}
& \Rightarrow \quad b a+a=a+b+a \\
& \Rightarrow \quad 0+a=a+b+a \quad \text { (since } \mathrm{b} a=0) \\
& \Rightarrow \quad a=a+b+a
\end{aligned}
$$

Therefore $(S,+)$ is a rectangular band.

Theorem 3.4: Let $(S,+,$.$) be a complemented semiring containing the multiplicative identity 1$. If $(S,$.$) is a$ band, then $(S,+)$ is a band.

Proof: Let $(S,+,$.$) is a complemented semiring and let ' 1$ ' be the multiplicative identity of $S$.

Since $S$ complemented semiring, wehave $a+b=1$ and $a b=b a=0$ forall $a, b \in S$.

Now consider $a+1+b=1$ and $a b=b a=0$ for all $a, b \in S$. (by theorem 3.1)

Multiplying with ' $a$ ' on both sides, we get

$$
\begin{aligned}
& a(a+1+b)=a 1 \\
\Rightarrow & a a+a 1+a b=a \\
\Rightarrow & a^{2}+a+a b=a \\
\Rightarrow & \left.a^{2}+a+0=a \quad \text { (since } a \mathrm{~b}=0\right) \\
\Rightarrow & a+a=a
\end{aligned}
$$

Therefore $(S,+)$ is a band.

Theorem 3.5: Let $(S,+,$.$) be a complemented semiring containing the multiplicative identity 1$. If $(S,+)$ is a band, then $S$ is multiplicative sub idempotent.

Proof: Let $(S,+,$.$) be a complemented semiring and let '1' be a multiplicative identity of S$.

We have $a+b=1$ and $a b=b a=0$ for all $a, b \in S$. 
Now consider $a+1+b=1$ and $a b=b a=0$ for all $a, b \in S$. (by theorem 3.1)

Multiplying with ' $a$ ' on both sides, we get

$$
\begin{aligned}
& a(a+1+b)=a 1 \\
\Rightarrow & a a+a 1+a b=a \\
\Rightarrow & a^{2}+a+a b=a \\
\Rightarrow & \left.a^{2}+a+0=a \quad \text { (since } a \mathrm{~b}=0\right) \\
\Rightarrow & a^{2}+a=a \\
\Rightarrow & a(a+1)=a \quad \text { (by theorem 3.1) } \\
\Rightarrow & a a=a \quad a^{2}=a
\end{aligned}
$$

Adding ' $a$ ' on both sides, we get

$$
\begin{aligned}
& \Rightarrow a+a^{2}=a+a \\
& \Rightarrow a+a^{2}=a \quad \text { (since }(S,+) \text { is a band) }
\end{aligned}
$$

Therefore $S$ is a multiplicative sub idempotent.

Theorem 3.6: Let ' $a$ ' is a complimented element in a semiring. Then $a^{n}+b^{n}=1$ for all $n \geq 1$.

Proof: Let ' $a$ ' be a complimented element in a $S$. Then there exists $b \in S$ such that

$$
a+b=1 \text { and } a b=b a=0 \text { for all } a, b \in S .
$$

Now consider $a+1+b=1$ and $a b=b a=0$ for all $a, b \in S$. (by theorem 3.1)

Squaring on both sides, we get

$$
\begin{gathered}
\Rightarrow \quad(a+1+b)^{2}=1^{2}=1 \\
\Rightarrow(a+1+b)(a+1+b)==1 \\
\Rightarrow \quad a^{2}+a+a b+a+1+b+b a+b+b^{2}=1 \\
\Rightarrow \quad a^{2}+a(1+b)+a+(1+b)+b(a+1)+b^{2}=1 \\
\Rightarrow \quad a^{2}+a b+a+b+b a+b^{2}=1 \quad(\text { by theorem 3.1) } \\
\Rightarrow a^{2}+a(b+1)+b(1+a)+b^{2}=1 \\
\Rightarrow a^{2}+a b+b a+b^{2}=1 \quad \text { (by theorem 3.1) } \\
\left.\Rightarrow a^{2}+0+0+b^{2}=1 \quad \text { (since } a \mathrm{~b}=\mathrm{b} a=0\right) \\
\Rightarrow a^{2}+b^{2}=1
\end{gathered}
$$

From (1) \& (2) we get

$$
\Rightarrow a^{2}+b^{2}=(a+1+b)^{2}
$$

Now consider $a+1+b=1$

Cubing on both sides, we get

$$
\begin{aligned}
\Rightarrow & (a+1+b)^{3}=1^{3}=1 \\
\Rightarrow & (a+1+b)^{2}(a+1+b)==1 \\
\Rightarrow & \left(a^{2}+b^{2}\right)(a+1+b)==1 \\
\Rightarrow & a^{3}+a^{2}+a^{2} b+b^{2} a+b^{2}+b^{3}==1 \\
\Rightarrow & a^{3}+a^{2}(1+b)+b^{2}(a+1)+b^{3}==1 \\
\Rightarrow & a^{3}+a^{2} b+b^{2} a+b^{3}==1 \quad \text { [from (3) ] } \\
\Rightarrow a^{3}+a a b+b b a+b^{3}==1 & \quad \text { (since } a \mathrm{~b}=\mathrm{b} a=0) \\
\Rightarrow & a^{3}+a 0+b 0+b^{3}==1 \\
\Rightarrow & a^{3}+0+0+b^{3}==1
\end{aligned}
$$

From (4) \& (5) we get 


$$
\begin{aligned}
& \Rightarrow a^{3}+b^{3}==(a+1+b)^{3} \\
\text { Also } & \Rightarrow(a+1+b)^{4}=1^{4}=1 \\
& \Rightarrow(a+1+b)^{3}(a+1+b)==1 \\
& \Rightarrow\left(a^{3}+b^{3}\right)(a+1+b)==1 \quad \text { from (6) } \\
& \Rightarrow a^{4}+a^{3}+a^{3} b+b^{3} a+b^{3}+b^{4}==1 \\
& \Rightarrow a^{4}+a^{3}(1+b)+b^{3}(a+1)+b^{4}==1 \\
& \Rightarrow a^{4}+a^{3} b+b^{3} a+b^{4}==1 \\
& \Rightarrow a^{4}+a^{2} a b+b^{2} b a+b^{4}==1 \\
& \Rightarrow a^{4}+a^{2} 0+b^{2} 0+b^{4}==1 \\
& \Rightarrow a^{4}+0+0+b^{4}==1 \\
& \Rightarrow a^{4}+b^{4}==1
\end{aligned}
$$

Continuing like this, we get.

Therefore $a^{n}+b^{n}=1$ for all $n \geq 1$.

\section{Conclusions}

In complemented semirings, the algebraic structures of a multiplicative semigroup $(S,$.$) determine$ the additive structure $(S,+)$ and vice versa.

In a complemented semiring $(S,+,$.$) , if 1$ is the multiplicative identity, then the multiplicative structure satisfies the band property. If the complemented semiring $(S,+,$.$) contains the multiplicative identity$ which is also an additive identity 1 , then the multiplicative structure satisfies the following properties: band, left (right) singulars, commutative, rectangular band and multiplicative sub idempotent.

\section{References}

[1] Arif Kaya and M. Satyanarayana - "Semi rings satisfying properties of distributive type". Proceeding of the American mathematical society, volume 82, number 3, July 1981

[2] Jonathan S. Golan - "semirings and their applications".

[3] Jonathan S. Golan - "semirings and Affine Equations over Them: Theory and Applications". Kluwer Academic Publishers (1999).

[4] M. Satyanarayana - "On the additive semi group of ordered semirings", semi group forum vol.31(1985), 193-199

[5] M.P. Grillet - "subdivision rings of a semiring". Fund. Math., vol.67 (1970), 67-74.

[6] J. Hanumanthachari and T. Vasanthi - "Structure of certain classes of semirings". Proc.of the First Internationals Symposium, Algebraic Structures and Number theory, Hong kong, August 8-3, (1988), 132-143. 